\title{
Establishment and Effects of Ginger and Kikyoto of a Haloperidol-Induced Dysphagia Model in Guinea Pigs
}

\author{
Takahiro Mizoguchi', Mitsue Ishisaka1, Yui Kobatake², Hiroaki Kamishina², \\ Yasuhiko Nishioka ${ }^{3}$, Tsukasa Kirimoto ${ }^{3}$, Masamitsu Shimazawa', Hideaki Hara ${ }^{{ }^{*}}$ \\ ${ }^{1}$ Molecular Pharmacology, Department of Biofunctional Evaluation, Gifu Pharmaceutical University, Gifu, Japan \\ ${ }^{2}$ Department of Veterinary Medicine, Faculty of Applied Biological Sciences, Gifu University, Gifu, Japan \\ ${ }^{3}$ Taiho Pharmaceutical Co., Ltd., Tokushima, Japan \\ Email: "hidehara@gifu-pu.ac.jp
}

Received 13 December 2015; accepted 19 February 2016; published 22 February 2016

Copyright (C) 2016 by authors and Scientific Research Publishing Inc.

This work is licensed under the Creative Commons Attribution International License (CC BY).

http://creativecommons.org/licenses/by/4.0/

c) (i) Open Access

\begin{abstract}
Dysphagia induces aspiration and causes aspiration pneumonia. There is no treatment for dysphagia fundamentally. Haloperidol reportedly induces dysphagia. In the present study, we established a haloperidol-induced dysphagia model in guinea pigs, and evaluated the effects of ginger, kikyoto, and a mixture of ginger and kikyoto on swallowing. Swallowing ability was evaluated using behavioral tests, computed tomography (CT), and videofluoroscopic examination of swallowing. To investigate the effect of ginger and kikyoto on swallowing, ginger, kikyoto, or a mixture of ginger and kikyoto was administered orally to guinea pigs with haloperidol-induced dysphagia. Effects of these compounds were evaluated with behavioral tests. Chronic administration of haloperidol reduced the number of swallows, as evaluated by the behavioral test and videofluoroscopic examination of swallowing. In our model, these compounds improved swallowing dysfunction. Our results suggest that this model might be useful in revealing the pathogenesis of dysphagia and evaluating compounds that might improve swallowing.
\end{abstract}

\section{Keywords}

Dysphagia, Guinea Pig, Videofluoroscopic Examination of Swallowing, Haloperidol, Ginger

\section{Introduction}

Dysphagia is the impairment of swallowing abilities, and often causes aspiration pneumonia [1]-[3]. Because "Corresponding author.

How to cite this paper: Mizoguchi, T., Ishisaka, M., Kobatake, Y., Kamishina, H., Nishioka, Y., Kirimoto, T., Shimazawa, M. and Hara, H. (2016) Establishment and Effects of Ginger and Kikyoto of a Haloperidol-Induced Dysphagia Model in Guinea Pigs. Pharmacology \& Pharmacy, 7, 97-105. http://dx.doi.org/10.4236/pp.2016.72013 
pneumonia is the leading cause of death in the elderly, it is necessary to improve dysphagia. Several compounds were suggested to improve dysphagia [4]-[6]; however, no available medicine treats dysphagia fundamentally. To date, few studies report about animal models of dysphagia [7]. Therefore, animal models of swallowing dysfunction are required to develop novel therapeutic drugs for the treatment of dysphagia.

It has been reported that dysphagia is induced by various diseases such as stroke, Parkinson's disease, and amyotrophic lateral sclerosis [8]-[11]. Recent studies revealed that dysphagia is present in up to 50\% of patients who suffered a stroke [12] [13]. Furthermore, antipsychotic drugs such as haloperidol and olanzapine also induce dysphagia [14] [15]. Administration of haloperidol, which is a dopamine D2 receptor antagonist, induces dysphagia clinically and impairs tongue functions in rats [14] [16].

In the present study, we established a dysphagia model in guinea pigs with chronic administration of haloperidol. Furthermore, we investigated swallowing dysfunction using behavioral tests and videofluoroscopy. Finally, we evaluated the effects of ginger (zingibers rhizome), which has a positive effect on swallowing in humans [17]. In addition to ginger, we evaluated kikyoto (containing platycodi radix and glycyrrhizae radix), which have the anti-allergic and anti-inflammatory actions [18] [19], and a mixture of ginger and kikyoto.

\section{Materials and Methods}

\subsection{Animals}

Male Hartley guinea pigs were used in the experiments. Guinea pigs were housed at $24^{\circ} \mathrm{C} \pm 2{ }^{\circ} \mathrm{C}$ under a $12: 12$ hr light:dark cycle (lights on from 8:00 to 20:00), and had ad libitum access to food and water. All animal care and treatment procedures were conformed to animal care guidelines of the Animal Experiment Committee of Gifu Pharmaceutical University. All efforts were made to minimize both suffering and the number of animals used.

\subsection{Generation of a Haloperidol-Induced Dysphagia Model}

Haloperidol was obtained from Wako (Osaka, Japan). It was dissolved in saline solution containing 1\% acetic acid. Haloperidol ( $1 \mathrm{mg} / \mathrm{kg}$, at volume of $1 \mathrm{ml} / \mathrm{kg}$ ) or vehicle (saline containing $1 \%$ acetic acid, at volume of 1 $\mathrm{ml} / \mathrm{kg}$ ) was administered subcutaneously twice a day for 15 days. The behavioral tests were performed on day 8 and 9. On days 13 and 15, swallowing ability was investigated with computed tomography (CT) and videofluoroscopy, respectively.

\subsection{Videofluoroscopic Examination of Swallowing}

Before the experiment, guinea pigs were fasting for $3.5 \mathrm{hr}$. The number of swallows was measured with videofluoroscopy after oral administration of various volumes of the contrast medium $(140,280,420,560$, and 700 $\mu$ l). The contrast medium contained barium sulfate and glucose in a ratio of 5:2, and was administered into the upper part of the pharynx. After administration, guinea pigs were placed into a box, and swallowing was recorded for 1 min using videofluoroscopic examination. Swallowing was defined as passage of the contrast medium from the pharynx to the stomach.

\subsection{Behavioral Test}

On days 8 and 9, the number of tongue movements was measured. Swallowing was induced by oral administration of distilled water $(400,500$, and $600 \mu \mathrm{l})$ into the upper part of the pharynx. Tongue movement was defined as the protrusion of the tongue. Tongue movements were measured for 1 min after the injection of distilled water based on visual inspection.

\subsection{Treatments in the Haloperidol-Induced Dysphagia Model}

Ginger emulsion and kikyoto were obtained from Taiho Pharmaceutical Co. Ltd. (Tokushima, Japan). They were suspended in $0.5 \%$ sodium carboxymethyl cellulose (CMC). Ginger emulsion (30 or $100 \mu \mathrm{l} / \mathrm{kg} / \mathrm{day}$ ), kikyoto (10 or $30 \mathrm{mg} / \mathrm{kg} / \mathrm{day})$, the mixture of ginger emulsion $(30 \mu \mathrm{l} / \mathrm{kg} / \mathrm{day})$ and kikyoto (10 mg/kg/day), or vehicle $(0.5 \% \mathrm{CMC})$ were administered orally at volume of $2 \mathrm{ml} / \mathrm{kg}$ for 7 days. On day 8 , behavioral tests were performed. 


\subsection{Statistical Analysis}

Data are presented as mean \pm standard error of the mean (S.E.M.). Statistical comparisons were performed using the Pearson's test for correlation, and the one-tailed Student's $t$-test and one- or two-sided Dunnett's tests for other statistics. We used the JSTAT software (Vector, Tokyo, Japan) or SPSS (IBM, Armonk, NY, USA) for the statistical analysis. Probability (p) values of less than 0.05 were considered statistically significant.

\section{Results}

\subsection{Chronic Administration of Haloperidol Impaired Swallowing, as Examined Using Videofluoroscopy}

We investigated the swallowing ability of the guinea pigs with videofluoroscopic examination. We evaluated frequency of the deglutition as the outcome measure of the ability of the deglutition as described previously [7].

The ratio of $\mathrm{BaSO}_{4}$ to glucose in the contrast medium was $5: 2(n=2)$.

We used a C-arm fluoroscope to perform the videofluoroscopic examination of swallowing (the equipment used is shown in Figure 1(a) and Figure 1(b)). Firstly, we administered several volumes of contrast medium orally, and measured the number of swallows in untreated guinea pigs. Delivery of the contrast medium from the pharynx to the stomach was observed with videofluoroscopy (Figure 1(c)). We defined movement of the contrast medium as "swallowing", and counted the number of swallows for 1 min. The number of swallows correlated with volume of the contrast medium (Table 1). Because the number of swallows was the highest after oral administration of $700 \mu \mathrm{l}$ contrast medium, we used this volume in the following examinations. On day 15 of the haloperidol injection, the number of swallows induced by oral administration of $700 \mu$ l contrast medium was measured for 1 min using videofluoroscopy $(n=9)$. The number of swallows in the haloperidol-treated group was significantly decreased compared with the control group (control: $5.78 \pm 0.86$, haloperidol-treated: $3.33 \pm$ 1.03) (Figure 1(d)). Sample movies of the swallows are available for the control (Supplementary Video (A)) and haloperidol-treated animals (Supplementary Video (B)).

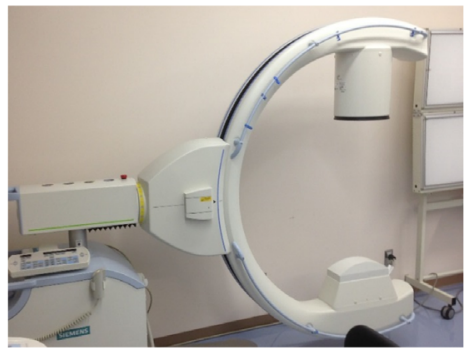

(a)

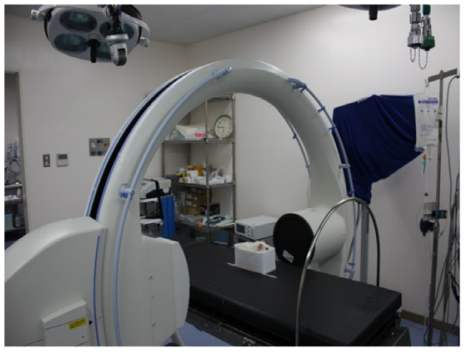

(b)

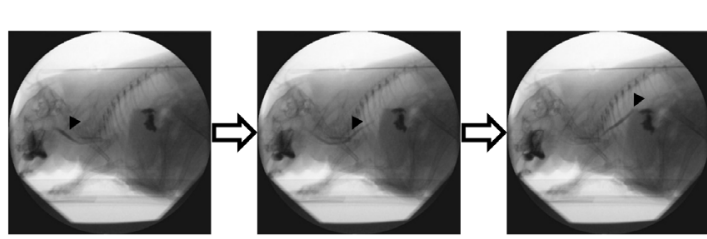

(c)

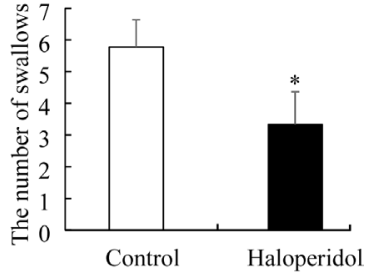

(d)

Figure 1. Videofluoroscopic examination of swallowing in haloperidol-treated guinea pigs. (a) The C-arm X-ray fluoroscopic machine used for the videofluoroscopic examination of swallowing; (b) Experimental setup for the videofluoroscopic examination of swallowing; (c) Representative photographs of swallowing recorded with videofluoroscopy. These photographs show the process of swallowing from left to right. Arrowheads indicate the contrast medium in the digestive system; (d) The number of swallows after the oral administration of $700 \mu$ contrast medium into the upper part of the pharynx in the control and haloperidol-treated animals. Values are expressed as mean \pm S.E.M. $(n=9)$; ${ }^{*} \mathrm{p}<0.05$, haloperidol-treated group vs. control group (onetailed Student's $t$-test). 
Table 1. The number of swallows induced by oral administration of the contrast medium measured with videofluoscopy.

\begin{tabular}{cccccc}
\hline Contract medium $(\mu \mathrm{l})$ & 140 & 280 & 420 & 560 & 700 \\
The number of swallowing & 3 & 4 & 4.5 & 6 & 7.5 \\
\hline
\end{tabular}

\subsection{Volume of Cntrast Medium in the Stomach and Intestinum Tenue Measured with CT}

Similarly, we investigated swallowing ability using CT (the equipment used for the experiment is shown in Figure S1(a) and Figure S1(b)). On day 13 of the haloperidol injections, we measured the volume of orally administered contrast medium in the stomach and small intestine $(n=9)$. There was no difference between the control and haloperidol-treated groups (Figure S1(c)).

\subsection{Administration of Distilled Water into the Upper Part of the Pharynx Induced a Swallowing Reflex}

In previous studies, swallowing ability was investigated by the oral administration of distilled water to guinea pigs [20] [21]. Here, we investigated whether distilled water induced a swallowing reflex. We administered 500 $\mu \mathrm{l}$ distilled water into the upper part of the pharynx of guinea pigs with a feeding needle. The protrusive action of the tongue was observed, and defined as "tongue movement" (Figure 2(a)). The number of tongue movements was measured for 1 min after the oral administration of distilled water $(n=4)$. The number of tongue movements in the group administered distilled water was significantly increased compared to the sham group (without administration) (Figure 2(b)). Tongue movements were not detected in the sham group. These results suggest that oral administration of distilled water induced tongue movements.

\subsection{Chronic Administration of Haloperidol Decreased the Number of Tongue Movements}

We investigated the number of tongue movements in the haloperidol-induced dysphagia model $(n=9)$. On day 8 and 9 , the number of tongue movements induced by the oral administration of three different volumes (400, 500, and $600 \mu \mathrm{l}$ ) of distilled water was measured for $1 \mathrm{~min}$. The number of tongue movements was significantly decreased in the haloperidol-treated group compared with the control group for all volumes (Figure 3(a)). Additionally, we observed a correlation between the number of swallows measured by videofluoroscopy and tongue movements induced by the oral administration of $600 \mu$ l distilled water $(r=0.4692, p<0.05)$ (Figure 3(b)), but not 400 or $500 \mu \mathrm{l}$ distilled water (Data not shown). These results suggest that chronic haloperidol treatment affects the movement of the tongue.

\subsection{Effects of Ginger and Kikyoto on Swallowing Action in the Haloperidol-Induced Dysphagia Model}

Finally, we evaluated the effect of ginger, which has a positive effect on swallowing in humans [17], on swallowing in the haloperidol-induced dysphagia model [control (vehicle and vehicle treated group), $\mathrm{n}=9$; haloperidol and vehicle treated group, $\mathrm{n}=8$; haloperidol and ginger ( $30 \mu \mathrm{l} / \mathrm{kg} / \mathrm{day})$ treated group, $\mathrm{n}=10$; haloperidol and ginger $(100 \mu \mathrm{l} / \mathrm{kg} /$ day) treated group, $\mathrm{n}=9$ ]. Ginger emulsion (ginger) was administrated orally to the haloperidol treated animals for 7 days. On day 8, the number of tongue movements induced by the oral administration of $600 \mu \mathrm{l}$ distilled water was measured for $1 \mathrm{~min}$. The number of tongue movements was significantly decreased in the haloperidol-treated group compared with the control group. Ginger $(100 \mu \mathrm{l} / \mathrm{kg} /$ day $)$, significantly ameliorated the reduced number of tongue movements induced by haloperidol (Figure 4(a)). In addition to the evalustion of the effect of ginger, the effect of kikyoto, or the mixture of ginger and kikyoto on swallowing was evaluated in the haloperidol-induced dysphagia model by the similar method [control (vehicle and vehicle treated group, $\mathrm{n}=12$; haloperidol and vehicle treated group, $\mathrm{n}=12$; haloperidol and kikyoto (10 m/kg/day) treated group, $n=9$; haloperidol and kikyoto (30 m/kg/day) treated group, $n=9$; haloperidol and the mixture treated group, $\mathrm{n}=8$. Kikyoto (30 mg/kg/day), and the mixture of ginger ( $30 \mu \mathrm{l} / \mathrm{kg} /$ day) and kikyoto (10 $\mathrm{mg} / \mathrm{kg} /$ day) significantly ameliorated the reduced number of tongue movements induced by haloperidol (Figure 4(b)). These results indicate that ginger and kikyoto may improve swallowing in the haloperidol-induced dysphagia model. 


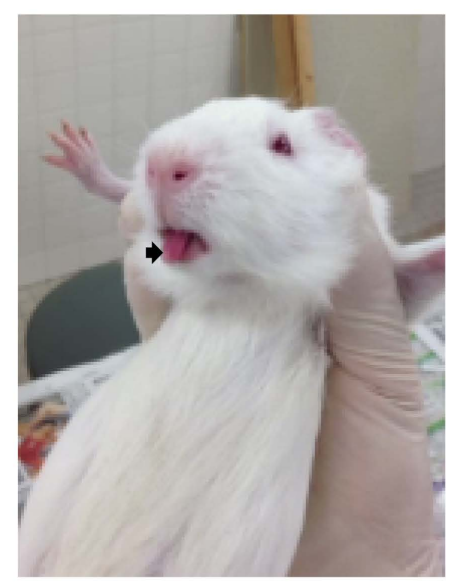

(a)

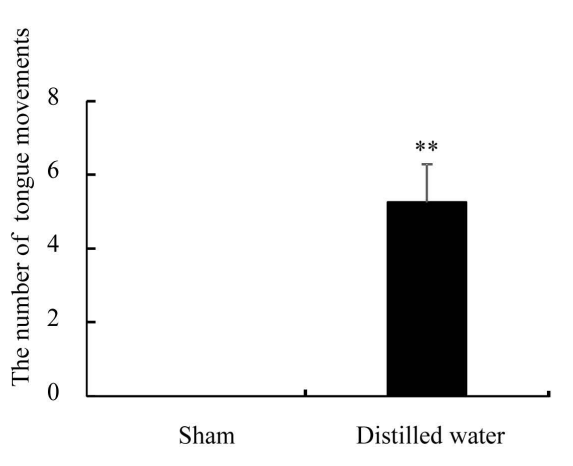

(b)

Figure 2. The tongue movement induced by distilled water. (a) Picture of the tongue movement. The arrow indicates the evaluated tongue movement; (b) The number of tongue movements in the sham group (no administration of distilled water) and in the animals administered with $500 \mu$ distilled water. Values are expressed as mean \pm S.E.M. $(n=4) ;{ }^{* *} \mathrm{p}<0.01$, distilled water administered group vs. sham group (two-tailed Student's $t$-test).

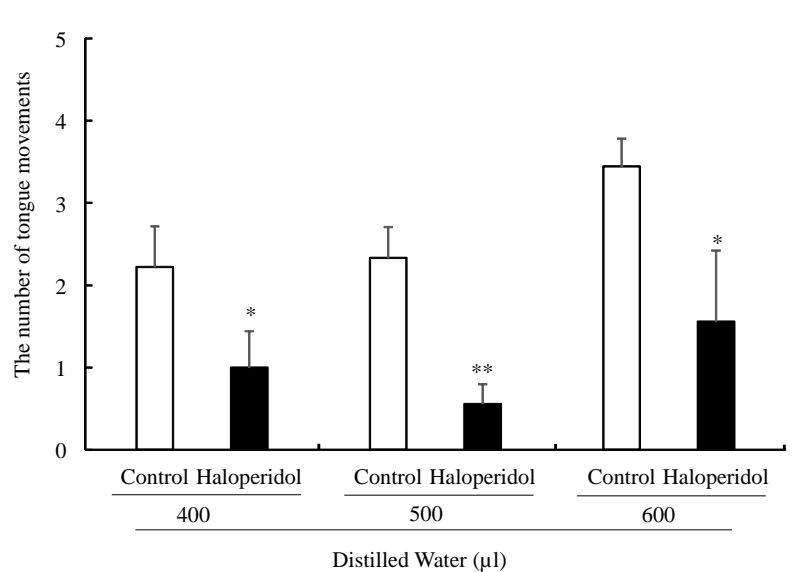

(a)

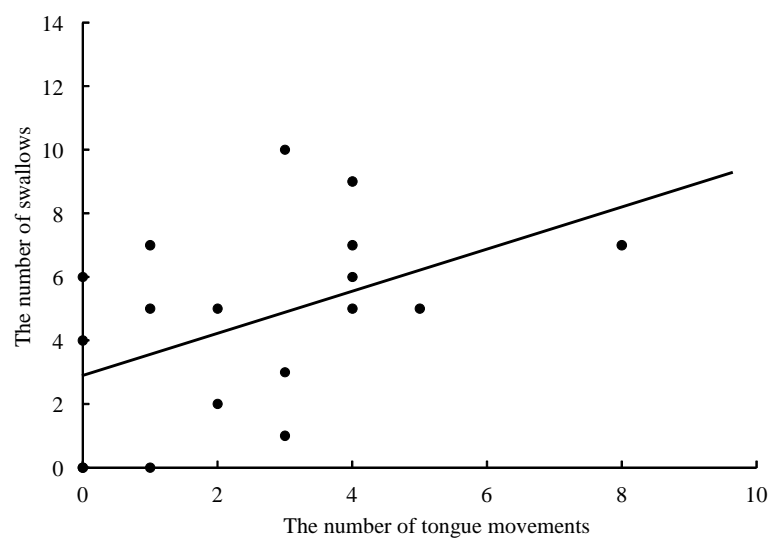

(b)

Figure 3. Change in the number of tongue movements in the haloperidol-treated guinea pigs. (a) The number of tongue movements induced by the oral administration of 3 different volumes $(400,500$, and $600 \mu \mathrm{l})$ of distilled water into the upper part of the pharynx in the control and haloperidol-treated groups. Values are expressed as mean \pm S.E.M. $(n=9)$, ${ }^{*} \mathrm{p}<0.05$ and ${ }^{* *} \mathrm{p}<0.01$, haloperidol-treated group vs. control group (one-tailed Student's $t$-test); (b) The correlation between the number of tongue movements induced by the oral administration of $600 \mu$ distilled water and the number of swallows measured with videofluoroscopy (Pearson's test).

\section{Discussion}

One of the main purposes of this study was to establish a dysphagia model using guinea pigs. Videofluoroscopy and behavioral tests were used to evaluate the swallowing ability of guinea pigs.

Videofluoroscopy is an effective clinical method to investigate the process of swallowing [22] [23]. Additionally, advice based on the results of videofluoroscopic examination of swallowing can help to avoid aspiration pneumonia [24]. To date, no studies have evaluated swallowing ability with videofluoroscopy in guinea pigs. In the present study, we showed that it is possible to evaluate the swallowing ability with videofluoroscopy using guinea pigs. It was observed that the number of swallows decreased in the haloperidol-treated guinea pigs. This suggests that chronic administration of haloperidol induces dysphagia in guinea pigs. However, there was no 


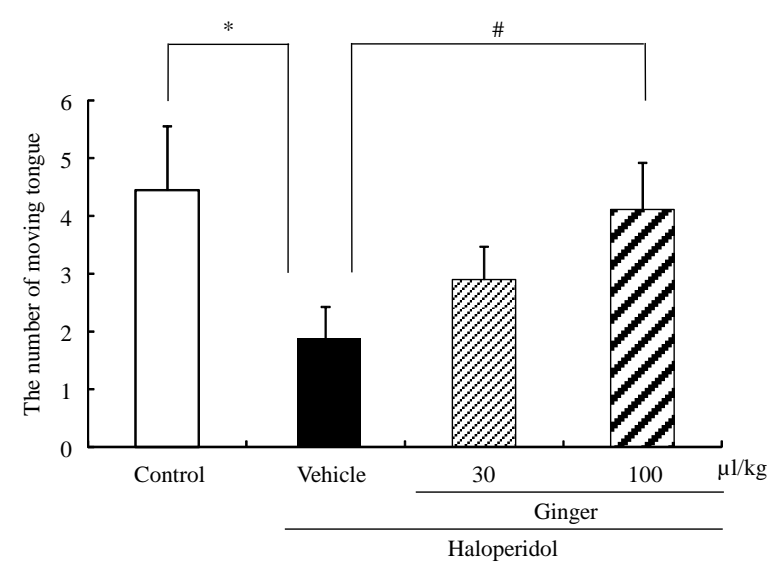

(a)

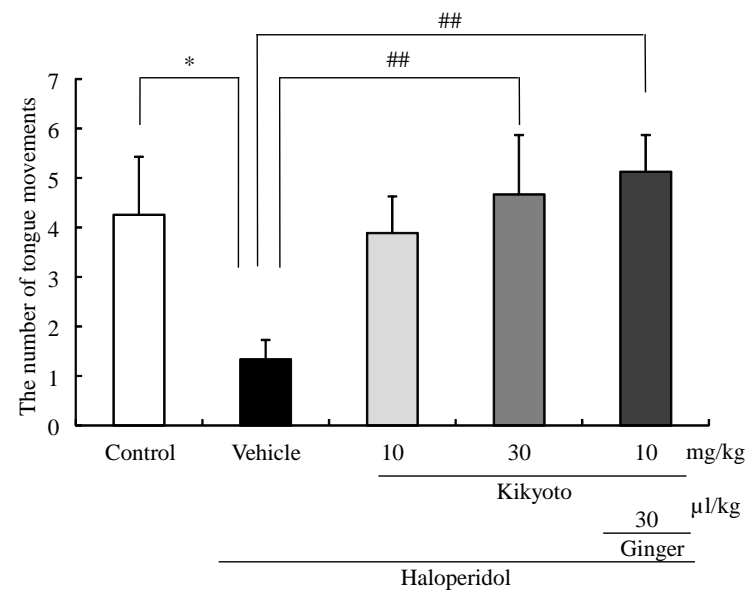

(b)

Figure 4. Effect of ginger and kikyoto on the haloperidol-induced dysphagia in guinea pigs. (a) The number of tongue movements induced by oral administration of $600 \mu \mathrm{l}$ distilled water into the upper part of the pharynx of guinea pigs. Values are expressed as mean \pm S.E.M.; control (vehicle and vehicle treated group), $n=9$; haloperidol and vehicle treated group, $\mathrm{n}=$ 8; haloperidol and ginger $(30 \mu \mathrm{l} / \mathrm{kg})$ treated group, $\mathrm{n}=10$; haloperidol and ginger $(100 \mu \mathrm{l} / \mathrm{kg})$ treated group, $\mathrm{n}=9 ;{ }^{*} \mathrm{p}<0.05$, haloperidol and vehicle treated group vs. the control group (one-tailed Student's t-test); ${ }^{\#} \mathrm{p}<0.05$, haloperidol and ginger $(100 \mu \mathrm{l} / \mathrm{kg}$ ) treated group vs. haloperidol and vehicle treated group (one-sided Dunnett's test); (b) The number of tongue movements induced by oral administration of $600 \mu \mathrm{l}$ distilled water into the upper part of the pharynx of guinea pigs. Values are expressed as mean \pm S.E.M.; control (vehicle and vehicle treated group), $\mathrm{n}=12$; haloperidol and vehicle treated group, $\mathrm{n}=$ 12; haloperidol and kikyoto (10 mg/kg) treated group, $\mathrm{n}=9$; haloperidol and kikyoto (30 mg/kg) treated group, $\mathrm{n}=9$; haloperidol and the mixture treated group, $\mathrm{n}=8 ;{ }^{*} \mathrm{p}<0.05$, haloperidol and vehicle treated group vs. control group (one-tailed Student's t-test); ${ }^{\#} \mathrm{p}<0.01$, haloperidol and kikyoto $(30 \mathrm{mg} / \mathrm{kg}$ ) or the mixture treated groups vs. haloperidol and vehicle treated group (two-sided Dunnett’s test).

difference in the volume of contrast medium in the stomach and small intestine of guinea pigs in the control and haloperidol-treated groups according to CT analysis. It is possible that the contrast medium was mixed with the fluids in the intestine and stomach; thus, amplifying the signal.

Additionally, we evaluated swallowing induced by the administration of distilled water into the upper part of the pharynx using a feeding needle. This behavioral test was evaluated based on visual observations; therefore, this method was easier to perform compared with the videofluoroscopic examination of swallowing. The tongue executes protrusive and retrusive actions during swallowing, and it has been reported that force of the protrusive action is reduced in old rats [25]. Therefore, we focused on the protrusive action of the tongue, and measured this behavior. The number of tongue movements in the haloperidol-treated group was significantly decreased compared with the control group. Additionally, we observed a correlation between the number of swallows measured by videofluoroscopy and the number of tongue movements (for $600 \mu l$ ). These results suggest that the number of tongue movements might be representative of swallowing ability.

Another purpose of the study was to evaluate the effects of ginger and kikyoto on the haloperidol-induced dysphagia in guinea pigs. Ginger contains numerous pungent phenolic compounds such as 6-gingerol, 6-shagol, 6-paradol, and zingerone [26]. Similar to capsaicin, 6-gingerols are vanilloid receptor 1 agonists [27]. Vanilloid receptor 1 regulates swallowing, and administration of capsaicin improves dysphagia in elderly people and rats with dysphagia induced by middle cerebral artery occlusion [7] [28] [29]. Additionally, it has been reported that gingerol promotes swallowing in young adult women [17]. In the present study, chronic administration of ginger emulsion $(100 \mu \mathrm{l} / \mathrm{kg} /$ day $)$ ameliorated the decrease in the number of tongue movements. Kikyoto is a crude drug containing platycodi radix and glycyrrhizae radix extracts. The active ingredient of platycodi radix is saponin and inulin. Glycyrrhizae radix contains glycyrrhizin and other compounds. Kikyoto is reported to have various effects, such as anti-allergic and anti-inflammatory actions; however, no reports implicate its effect in dysphagia [18] [19]. In our animal model, chronic administration of kikyoto (30 mg/kg/day) increased the number of tongue movements, suggesting that kikyoto might improve dysphagia. Further examinations are necessary to reveal its mechanism of action in dysphagia. Finally, the mixture of ginger and kikyoto improved swallowing dysfunc- 
tion at doses that were not efficient individually. This finding indicates that ginger and kikyoto might have different mechanisms of action and thus their effects are additive.

\section{Conclusion}

In conclusion, chronic administration of haloperidol induced dysphagia in guinea pigs, and ginger and kikyoto were effective for alleviating swallowing dysfunction in this model. Therefore, our model might be useful to clarify the pathogenesis of dysphagia and to test compounds that might improve swallowing.

\section{Acknowledgements}

We thank Mr. Eiji Naito, and Miss. Kanae Oyake (Department of Veterinary Medicine, Faculty of Applied Biological Sciences, Gifu University, Gifu, Japan) for technical support.

\section{Disclosure Statement}

Prof. Hideaki Hara has received a research grant from Taiho Pharmaceutical Co. Ltd. (Tokushima, Japan). The other authors declare that have no conflict of interest.

\section{References}

[1] Martin, B.J., Corlew, M.M., Wood, H., Olson, D., Golopol, L.A., Wingo, M. and Kirmani, N. (1994) The Association of Swallowing Dysfunction and Aspiration Pneumonia. Dysphagia, 9, 1-6. http://dx.doi.org/10.1007/BF00262751

[2] Taniguchi, M.H. and Moyer, R.S. (1994) Assessment of Risk Factors for Pneumonia in Dysphagic Children: Significance of Videofluoroscopic Swallowing Evaluation. Developmental Medicine \& Child Neurology, 36, 495-502. http://dx.doi.org/10.1111/j.1469-8749.1994.tb11879.x

[3] Harkness, G.A., Bentley, D.W. and Roghmann, K.J. (1990) Risk Factors for Nosocomial Pneumonia in the Elderly. American Journal of Medicine, 89, 457-463. http://dx.doi.org/10.1016/0002-9343(90)90376-O

[4] Ohkubo, T., Chapman, N., Neal, B., Woodward, M., Omae, T. and Chalmers, J. (2004) Effects of an Angiotensin Converting Enzyme Inhibitor-Based Regimen on Pneumonia Risk. American Journal of Respiratory and Critical Care Medicine, 169, 1041-1045. http://dx.doi.org/10.1164/rccm.200309-1219OC

[5] Shinohara, Y. (2006) Antiplatelet Cilostazol Is Effective in the Prevention of Pneumonia in Ischemic Stroke Patients in the Chronic Stage. Cerebrovascular Diseases, 22, 57-60. http://dx.doi.org/10.1159/000092922

[6] Nakagawa, T., Wada, H., Sekizawa, K., Arai, H. and Sasaki, H. (1999) Amantadine and Pneumonia. Lancet, $353,1157$. http://dx.doi.org/10.1016/S0140-6736(98)05805-X

[7] Sugiyama, N., Nishiyama, E., Nishikawa, Y., Sasamura, T., Nakade, S., Okawa, K., Nagasawa, T. and Yuki, A. (2014) A Novel Animal Model of Dysphagia Following Stroke. Dysphagia, 29, 61-67. http://dx.doi.org/10.1007/s00455-013-9481-x

[8] Daniels, S.K., Brailey, K., Priestly, D.H., Herrington, L.R., Weisberg, L.A. and Foundas, A.L. (1998) Aspiration Inpatients with Acute Stroke. Archives of Physical Medicine and Rehabilitation, 79, 14-19. http://dx.doi.org/10.1016/S0003-9993(98)90200-3

[9] Smithard, D.G., O’Neill, P.A., Park, C., England, R., Renwick, D.S., Wyatt, R., Morris, J. and Martin, D.F. (1998) Canbedside Assessment Reliably Exclude Aspiration Following Acute Stroke? Age Ageing, 27, 99-106. http://dx.doi.org/10.1093/ageing/27.2.99

[10] Ali, G.N., Wallace, K.L., Schwartz, R., DeCarle, D.J., Zagami, A.S. and Cook, I.J. (1996) Mechanisms of Oralpharyngeal Dysphagia in Patients with Parkinson’s Disease. Gastroenterology, 110, 383-392. http://dx.doi.org/10.1053/gast.1996.v110.pm8566584

[11] Ertekin, C., Aydogdu, I., Yuceyar, N., Kiylioglu, N., Tarlaci, S. and Uludag, B. (2000) Pathophysiological Mechanisms of Oropharyngeal Dysphagia in Amyotrophic Lateral Sclerosis. Brain, 123, 125-140. http://dx.doi.org/10.1093/brain/123.1.125

[12] Palmer, J.B., Drennan, J.C. and Baba, M. (2000) Evaluation and Treatment of Swallowing Impairments. American Family Physician, 61, 2453-2462.

[13] Smithard, D.G., O’Neill, P.A., England, R.E., Park, C.L., Wyatt, R., Martin, D.F. and Morris, J. (1997) The Natural History of Dysphagia Following a Stroke. Dysphagia, 12, 188-193. http://dx.doi.org/10.1007/PL00009535

[14] Nagamine, T. (2008) Serum Substance P Levels in Patients with Chronic Schizophrenia Treated with Typical or Atypical Antipsychotics. Neuropsychiatric Disease and Treatment, 4, 289-294. http://dx.doi.org/10.2147/NDT.S2367 
[15] Sagar, R., Varghese, S.T. and Balhara, Y.P. (2005) Olanzapine-Induced Double Incontinence. Indian Journal of Medical Sciences, 59, 163-164. http://dx.doi.org/10.4103/0019-5359.16123

[16] Ciucci, M.R. and Connor, N.P. (2009) Dopaminergic Influence on Rat Tongue Function and Limb Movement Initiation. Experimental Brain Research, 194, 587-596. http://dx.doi.org/10.1007/s00221-009-1736-2

[17] Krival, K. and Bates, C. (2012) Effects of Club Soda and Ginger Brew on Lingua-Palatal Pressures in Healthy Swallowing. Dysphagia, 27, 228-239. http://dx.doi.org/10.1007/s00455-011-9358-9

[18] Oh, Y.C., Kang, O.H., Choi, J.G., Lee, Y.S., Brice, O.O., Jung, H.J., Hong, S.H., Lee, Y.M., Shin, D.W., Kim, Y.S. and Kwon, D.Y. (2011) Anti-Allergic Activity of a Platycodon Root Ethanol Extract. International Journal of Molecular Sciences, 11, 2746-2758. http://dx.doi.org/10.3390/ijms11072746

[19] Parihar, M., Chouhan, A., Harsoliya, M.S., Pathan, J.K., Banerjee, S., Khan, N. and Patel, V.M. (2011) A ReviewCough \& Treatments. International Journal of Natural Products Research, 1, 9-18.

[20] Jin, Y., Sekizawa, K., Fukushima, T., Morikawa, M., Nakazawa, H. and Sasaki, H. (1994) Capsaicin Desensitization Inhibits Swallowing Reflex in Guinea Pigs. American Journal of Respiratory and Critical Care Medicine, 149, 261263. http://dx.doi.org/10.1164/ajrccm.149.1.7509247

[21] Jia, Y.X., Sekizawa, K., Ohrui, T., Nakayama, K. and Sasaki, H. (1998) Dopamine D1 Receptor Antagonist Inhibits Swallowing Reflex in Guinea Pigs. American Journal of Physiology, 274, R76-R80.

[22] Holas, M.A., De Pippo, K.L. and Reding, M.J. (1994) Aspiration and Relative Risk of Medical Complications Following Stroke. Archives of Neurology, 51, 1051-1053. http://dx.doi.org/10.1001/archneur.1994.00540220099020

[23] Teasell, R.W., McRae, M., Marchuk, Y. and Finestone, H.M. (1996) Pneumonia Associated with Aspiration Following Stroke. Archives of Physical Medicine and Rehabilitation, 77, 707-709. http://dx.doi.org/10.1016/S0003-9993(96)90012-X

[24] Cappabianca, S., Reginelli, A., Monaco, L., Del Vecchio, L., Di Martino, N. and Grassi, R. (2008) Combined Video Fluoroscopy and Manometry in the Diagnosis of Oropharyngeal Dysphagia: Examination Technique and Preliminary Experience. La Radiologia Medica, 113, 923-940. http://dx.doi.org/10.1007/s11547-008-0290-5

[25] Nagai, H., Russell, J.A., Jackson, M.A. and Connor, N.P. (2008) Effect of Aging on Tongue Protrusion Forces in Rats. Dysphagia, 23, 116-121. http://dx.doi.org/10.1007/s00455-007-9103-6

[26] Surh, Y.J., Lee, E. and Lee, J.M. (1998) Chemoprotective Properties of Some Pungent Ingredients Present in Redpepper and Ginger. Mutation Research, 402, 259-267. http://dx.doi.org/10.1016/S0027-5107(97)00305-9

[27] Dedov, V.N., Tran, V.H., Duke, C.C., Connor, M., Christie, M.J., Mandadi, S. and Roufogalis, B.D. (2002) Gingerols: A Novel Class of Vanilloid Receptor (VR1) Agonists. British Journal of Pharmacology, 137, 793-798. http://dx.doi.org/10.1038/sj.bjp.0704925

[28] Ebihara, T., Takahashi, H., Ebihara, S., Okazaki, T., Sasaki, T., Watando, A., Nemoto, M. and Sasaki, H. (2005) Capsaicin Troche for Swallowing Dysfunction in Older People. Journal of the American Geriatrics Society, 53, 824-828. http://dx.doi.org/10.1111/j.1532-5415.2005.53261.x

[29] Watando, A., Ebihara, S., Ebihara, T., Okazaki, T., Takahashi, H., Asada, M. and Sasaki, H. (2004) Effect of Temperature on Swallowing Reflex in Elderly Patients with Aspiration Pneumonia. Journal of the American Geriatrics Society, 52, 2143-2144. http://dx.doi.org/10.1111/j.1532-5415.2004.52579 3.x 


\section{Supplementary Material and Methods}

\section{Computed Tomography (CT)}

On day 13, CT was performed. Before the experiment, guinea pigs were fasting for $12 \mathrm{hr}$. The contrast medium used for the experiment consisted of $500 \mu \mathrm{l}$ of barium sulfate and $200 \mu \mathrm{l}$ of glucose. Guinea pigs were placed in the box afteroral administration of the contrast medium. CT images were exposed at 1, 3, 5, 9, and 15 min after the administration. The volume of orally administered contrast medium in the stomach and small intestine was measured with OsiriX (OsiriX Foundation, Geneva, Switzerland).

\section{Supplementary Video}

\section{Sample Movies of Videofluoroscopic Examination of Swallowing}

(A) A sample movie of control group. https://drive.google.com/file/d/0B0bQSsm7L31aNTRuRDZ1WTNTT1k/view?usp=sharing

(B) A sample movie of haloperidol-treated group.

https://drive.google.com/file/d/0B0bQSsm7L31ab2RLbXBLdmI1Zm8/view?usp=sharing

The number of swallows in haloperidol-treated group was significantly decreased with compared with control group.

\section{Supplementary Figure}

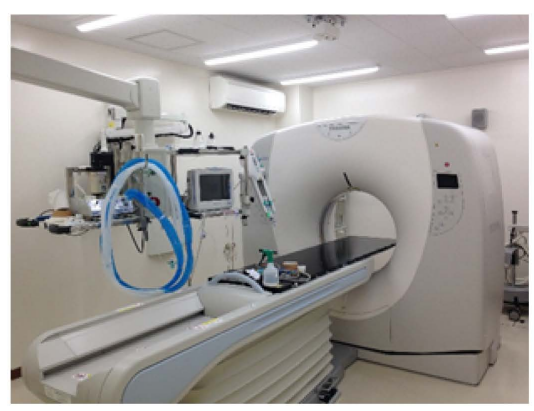

(a)

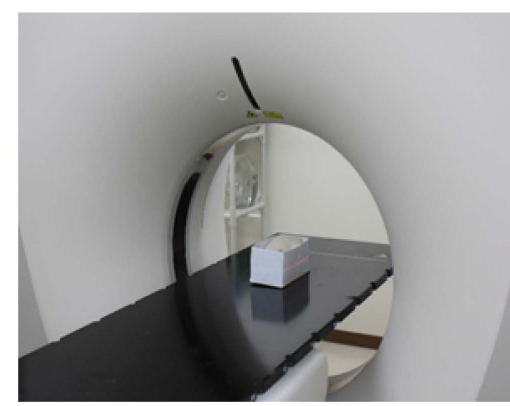

(b)

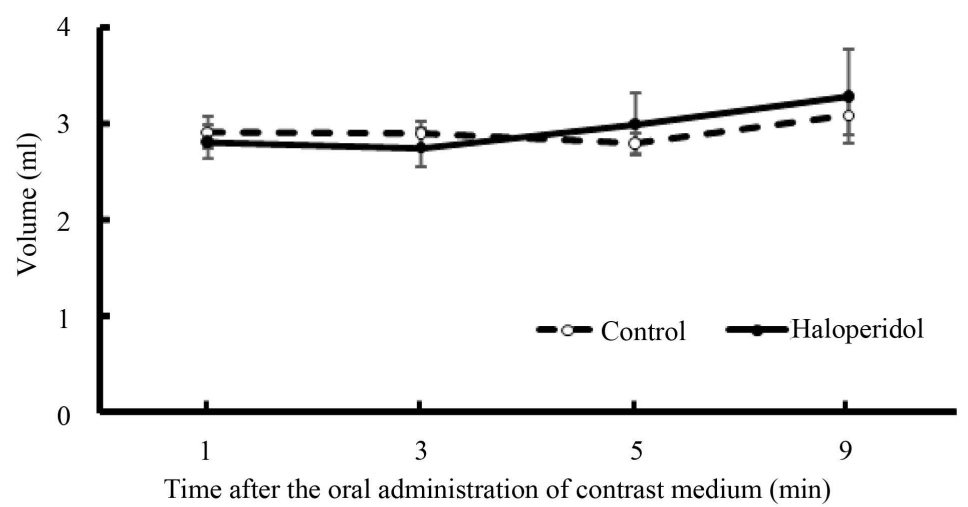

(c)

Figure S1. CT in haloperidol-treated guinea pigs. (a) CT machine used for computed tomography (CT); (b) Experimental setup for CT; (c) The volume of orally administered contrast medium in stomach and small intestine. Values are expressed as the mean \pm S.E.M. $(n=9)$. 